COLLOQUE DE PHYSIQUE

Colloque C2, supplément au $\mathrm{n}^{\circ} 2$, Tome 51, Février 1990

ler Congrès Français d'Acoustique 1990

\title{
LE BRUIT ম̀ BORD DES NAVIRES À PASSAGERS, MÉTHODE DE CALCUL PRÉVISIONNEL - CORRELATTION CALCULS - MESURES
}

A. BLANChet et G. CHAtel*

Chantiers de 1'Atlantique, BP. 400, F-44608 Saint-Nazaire, France * SECAV (Société Etude et Contrôle en Acoustique et Vibration), 6 , Boulevard de Brazza, F-13008 Marseille, France

Résumé : Les actions entreprises par le Chantier pour satisfaire aux critères de bruit dans les emménagements passagers des navires de croisières sont présentées et décrites.

La prévision des niveaux sonores dans les emménagements des navires est basée sur une méthode qui consiste à :

- modéliser les sources de bruit de l'appareil propulsif (moteurs, hélices),

- déterminer les transferts de vibrations de structure,

- quantifier la puissance acoustique rayonnée par les diffêrents éléments constituant une cabine.

Les calculs prévisionnels sont ensuite comparés aux mesures effectuées à bord sur deux navires à passagers.

Abstract : The studies and the disposals provided by the Yard to satisfy the noise requirements in the passenger accomodation in cruise ships are discribed in this paper.

The noise prediction in the accomodation of passenger ships is based on the following method:

- modelize the noise sources as the main engines, propellers;

- determine the structural transferts,

- quantify the acoustical power radiated by the each components of a cabin.

At the end, the results of the noise levels calculated are compared with the noise levels measured on board of two passenger ships.

\section{- 1 - Introduction}

Sur un navire de croisière, le confort offert aux passagers est de première importance. Les armateurs exigent dans leur spécification des niveaux sonores compatibles avec le confort attendu par leur clientèle.

Les dispositions prises par le chantier pour réduire le bruit transmis dans les emménagements, et plus particulièrement dans les cabines passagers, sont basées sur des études de prévision des niveaux sonores. Compte tenu des coûts importants engendrés par les dispositifs d'isolation et les pénalités liées aux niveaux sonores contractuels, il est nécessaire de déterminer avec précision les données de base du programme prévisionnel et tout particulièrement la modèlisation des sources de bruit et la quantification des transferts dans les structures du navire. 
- 2 - Spécification des navires de croisières - Critères de bruit -

Comme il n'existe aucune recommandation internationale pour les navires de croisières, les armateurs exigent des niveaux sonores de plus en plus stricts dans les emménagements réservés aux passagers. Par contre, le code international IMO A 468 Xil spécifie des niveaux sonores limites de $60 \mathrm{~dB}(\mathrm{~A})$ pour les cabines d'équipage. Le tableau montre l'évolution depuis 1983, des niveaux sonores exigés par les armateurs.

- Spécification Armateurs

$\begin{array}{lccc}\text { LOCAUX } & \text { Paquebot (A) } & \text { Paquebot (B) } & \text { Paquebot (C) } \\ \text { PASSAGERS } & \text { construit en 1983-84 } & \text { construit en 86-87 } & \text { construit en 88-89 } \\ \text { CABINES } & 60 \mathrm{~dB}(\mathrm{~A}) & 45 \mathrm{~dB}(\mathrm{~A}) & 50 \mathrm{~dB}(\mathrm{~A}) \\ \text { Locaux Publics } & 65 \mathrm{~dB}(\mathrm{~A}) & 55 \mathrm{~dB}(\mathrm{~A}) & 55 \mathrm{~dB}(\mathrm{~A})\end{array}$

- 3 - Actions sur les principales sources de bruit et prévision des niveaux sonores

Pour satisfaire les niveaux sonores de 45 ou $50 \mathrm{~dB}(A)$ dans les cabines de paquebot, le Chantier a entrepris des actions afin de réduire les sources principales de bruit. Les moteurs de propulsion sont montés sur suspensions élastiques, ce qui implique la mise en place d'accouplements élastiques entre réducteurs et moteurs. Ces moteurs sont, en général des moteurs semi-rapides, de poids de l'ordre de $100 \mathrm{~T}$ et dont la puissance développée varie de 4800 à $5500 \mathrm{KW}$. Les groupes électrogènes, considérés également comme une source principale de bruit sont montés sur suspensions élastiques.

La minimisation des fluctuations de pression et de la cavitation générées par les hélices fait l'objet d'études géomètriques et hydrodynamiques préalables.

- 4 - Prévision du bruit othélice

La prévision du bruit transmis dans les emménagements du navire nécessite ơune part, la détermination de la puissance de cette source et d'autre part, les transferts entre la source hélice et les vibrations sur les ponts du navire.

- Quantification de la source hélice : La source hélice est modélisée par un monopole dont le débit de vitesse volumique est déterminé par une méthode semi-empirique $/ 1 /$ prenant en considération un nombre important de paramètres hydrodynamiques et géomètriques.

Le niveau de débit de vitesse volumique du bruit de l'hélice, exprimé en décibel, est donné par bande de tiers d'octave, par la formule suivante :

$$
L U(i)=c_{1 j} \cdot\left(X^{\prime} Q\right)+c_{2 j} \cdot F+c_{3 j}+20 \lg \left(n \cdot D_{p}{ }^{3}\right)-20 \lg (n \cdot 2)
$$

avec: $D_{p}$ : diamètre de I'hélice $\quad Z \quad$ nombre de pales

$$
\mathrm{n} \text { : vitesse de rotation } \quad \mathrm{F} \text { : paramètre géométrique adimensionel }
$$

$K^{\prime} \mathrm{Q}$ : coefficient fonction du couple

La bande de tiers d'octave $i$ est déterminée à partir de la fréquence de passage des pales j

$$
j=i+1-(10 \log (N Z / 60)+0,5)
$$

Résultats : Les hélices d'un paquebot ont fait l'objet d'une part d'un calcul prévisionnel en utilisant la formule (1) et d'autre part, de mesure de bruit rayonné dans l'eau, le paquebot étant à vitesse et puissance stabilisées. On observe, sur la figure 1, une bonne concordance entre les résultats du calcul et la mesure. 


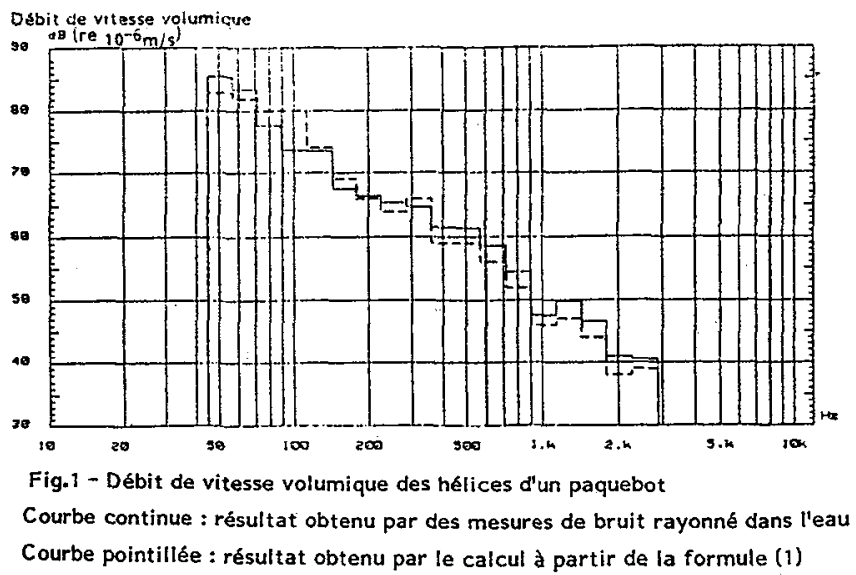

- Transfert du bruit d'élice : Le transfert entre le bruit de l'hélice et les vibrations sur les structures du navire est déterminé expérimentalement par des mesures faisant appel au principe de réciprocité. II est considéré un réseau hétérogène à deux pôles, mécanique-acoustique, et la relation particulière du théorème prise en considération est la suivante :

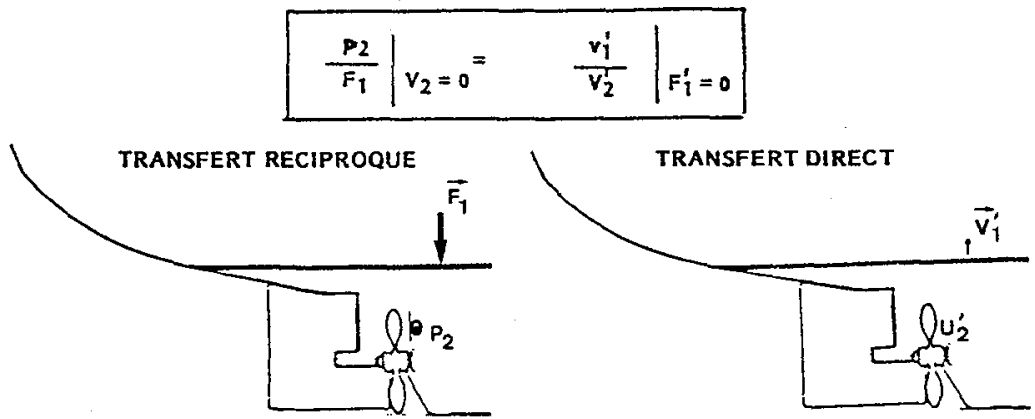

Fig.2 - Procédure pour la mesure des fonctions de transfert entre le débit de vitesse volumique de la source hélice et la vitesse vibratoire de la structure du navire.

\section{- 5 - Prévision du bruit des moteurs principaux}

Les vibrations de structure, induites sur les différents ponts du navire par le fonctionnement des moteurs principaux, sont quantifiées respectivement pour une excitation mécanique aux points de liaison, et pour une excitation acoustique par le bruit aérien. Comme pour les sources. hélices, il est utilisé une méthode de réciprocité pour quantifier le transfert entre la force équivalente des moteurs principaux montés sur suspensions élastiques et les vibrations de structure transmises sur les ponts. Des mesures acoustiques permettent également, de quantifier la part du transfert des bruits aériens dans les vibrations de structure.

\section{- 6 - Puissances acoustiques partielles rayonnées par les éléments de cabine}

Pour déterminer les puissances acoustiques partielles rayonnées par les différents éléments d'une cabine soumise à des vibrations de structure, il a été réalisé un système de mesures intensimétriques sur un banc d'essais. Deux cabines sont montées à l'intérieur d'une structure métallique de construction standardisée simulant deux ponts et le bordé. Un transducteur électrodynamique excite l'ensemble de la structure métallique. 
La répartition des vitesses vibratoires sur les différentes structures métalliques du banc d'essais sont représentatives des distributions des vitesses vibratoires à bord sur les deux ponts superposés. Des séries de mesures intensimétriques sont effectuées systématiquement sur les éléments caractéristiques des cabines, sols, cloisons et plafonds. La puissance acoustique partielle rayonnée par l'élément de cabine est exprimée en fonction de la vitesse vibratoire moyenne du pont inférieur. La figure 3 présente l'intensité moyenne rayonnée par le sol de la cabine avec et sans sol flottant, en fonction des vibrations de structure du pont.
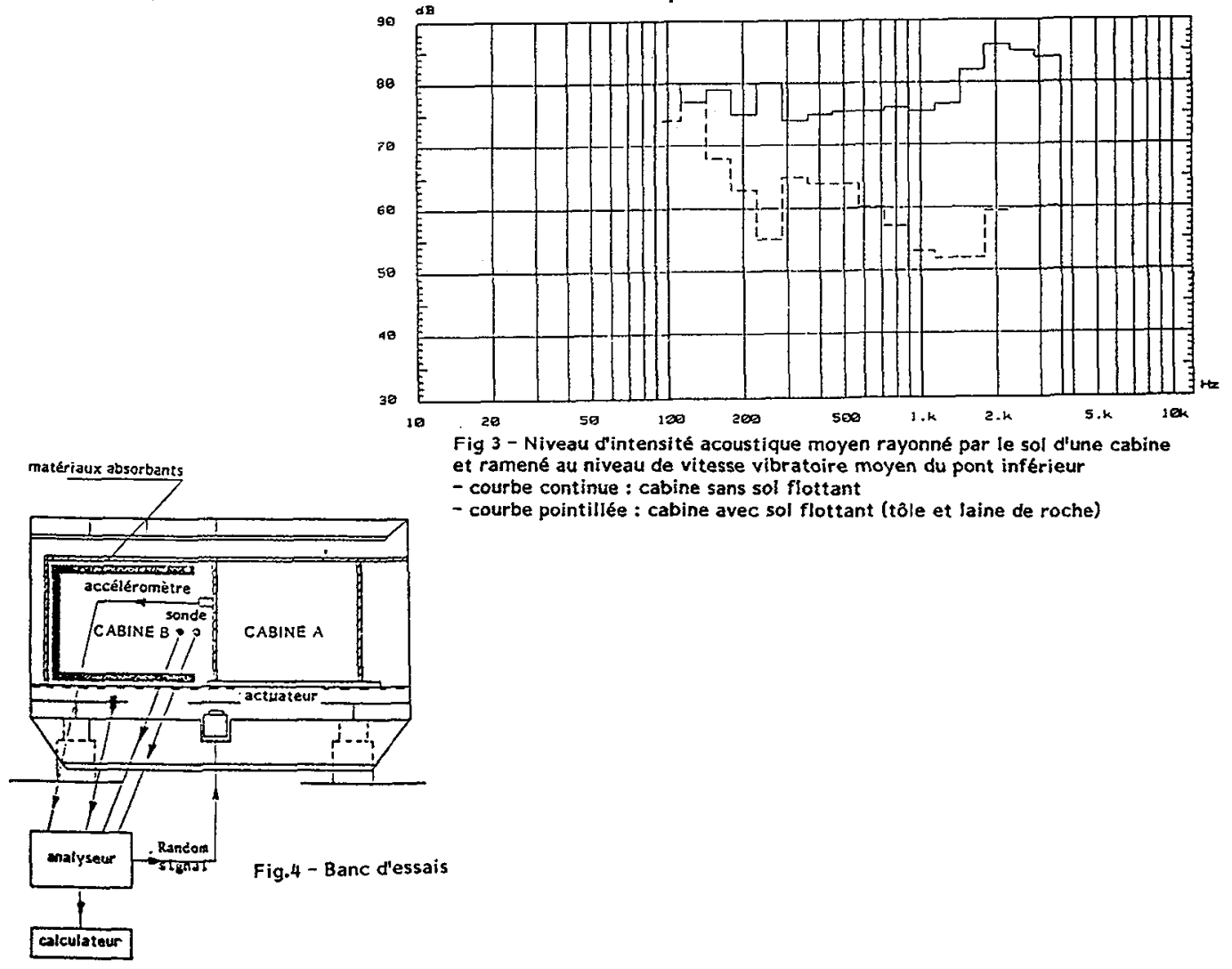

\section{- 7- Conclusion}

Ces études de prévision des niveaux sonores ont été appliquées sur plusieurs navires à passagers. Les résultats des mesures à bord ont montré que lincertitude sur les niveaux prévisionnels n'excédait pas $3 \mathrm{~dB}(\mathrm{~A})$ dans les emménagements dont le niveau sonore était directement influencé par le bruit des sources principales (hélices, moteurs de propulsion).

\section{REFERENCES}

11/ A. DE BRUIJN, W.H. MOELKER, F.C.J. ABSIL, "Prediction method for the acoustic source strength of propeller cavitation" - ISSA 86 -

121 J.W. VERHEIJ, "Multi-path sound transfer from resiliently mounted shipboard machinery" Thesis Delft, 1982

13/ A. BLANCHET, G.CHATEL, A. PARADIS, "Study of structure-borne noise transmission inside cabins by sound-intensity measurements" - ISSA 86 - 